\title{
The impact of Pre-Processing on the Classification of MEDLINE Documents
}

\author{
Carlos Adriano Gonçalves ${ }^{1}$, Célia Talma Gonçalves ${ }^{2,3}$, Rui Camacho ${ }^{1}$, Eugénio \\ Oliveira $^{2}$ \\ 1 LIAAD \& FEUP - Faculdade de Engenharia da Universidade do Porto \\ 2 LIACC \& FEUP - Faculdade de Engenharia da Universidade do Porto \\ 3 CEISE \& ISCAP - Instituto Superior de Contabilidade e Administração do Porto \\ \{carlos.adriano, talma, rcamacho, eco\}@fe.up.pt
}

\begin{abstract}
The amount of information available in the MEDLINE database makes it very hard for a researcher to retrieve a reasonable amount of relevant documents using a simple query language interface. Automatic Classification of documents may be a valuable technology to help reducing the amount of documents retrieved for each query. To accomplish this process it is of capital importance to use appropriate pre-processing techniques on the data. The main goal of this study is to analyse the impact of pre-processing techniques in text Classification of MEDLINE documents. We have assessed the effect of combining different pre-processing techniques together with several classification algorithms available in the WEKA tool. Our experiments show that the application of pruning, stemming and WordNet reduces significantly the number of attributes and improves the accuracy of the results.
\end{abstract}

Key words:Text Classification, Machine Learning, Pre-Processing, Information Retrieval, MEDLINE

\section{Introduction}

Molecular biology and biomedicine scientific publications are available (at least the abstracts) in Medical Literature Analysis and Retrieval System On-line (MEDLINE). MEDLINE is the U.S. National Library of Medicine's (NLM) premier bibliographic database that contains over 16 million references to journal articles in life sciences with a concentration on biomedicine. A distinctive feature of MEDLINE is that the records are indexed with NLM's Medical Subject Headings (MeSH terms). MEDLINE is the major component of PubMed [1], a database of citations of the National Library of Medicine in the United States. PubMed comprises more than 19 million citations for biomedical articles from MEDLINE and life science journals. The result of a MEDLINE/PubMed search is a list of citations ${ }^{4}$ to journal articles. The results of such search is, quite often,

${ }^{4}$ including authors, title, journal name, the abstract of the paper, keywords and MeSH terms 
a huge amount of documents, making it very hard for researchers to efficiently reach the most relevant documents for their queries. As this is a very relevant and actual topic of investigation we investigate the use of Machine Learning-based text classification techniques to help in the identification of a reasonable amount of relevant documents in MEDLINE. In this study we will focus on assessing the effect of different pre-processing techniques in the quality of classifiers available in the Weka tool.

The rest of this paper is structured as follows. In Section 2 we overview the pre-processing techniques used. In Section 3 we present the text classification problem and existing techniques. We report on related work in Section 4. Section 5 describes the experiments and Section 6 the results obtained. Section 7 concludes the paper presenting the conclusions and future work.

\section{Pre-Processing Techniques}

Before applying any data analysis technique it is necessary to pre-process the collection of documents. In our study we have used the following pre-processing techniques:

- Tokenization, the process of breaking a text into tokens. A token is a nonempty sequence of characters, excluding spaces and punctuation.

- Lowercase conversion converts all terms into lower cases.

- Special character removal removes all the special characters $(+,-$, !, ?, $.,,, ;,:,\{\},,=, \&, \#, \%, \$,[],, /,<,>, \backslash, ", ", \mid)$ and digits.

- Stop Word Removal removes words that are meaningless such as articles. conjunction and prepositions (e.g., a, the, at, etc.). These words are meaningless for the evaluation of the document content.

- Stemming is a widely used technique in text analysis. Stemming is the process of removing inflectional affixes of words reducing the words to their stem.

- Pruning discards terms either appearing rarely or "too frequently". Terms that rarely appear in a document or terms that appear too frequently do not contribute to identify the topic of the document. The most common techniques are term frequency and document frequency.

- Treating synonyms: the possibility to take care of synonyms may be seen as another pre-processing technique. If two words or terms mean the same thing, e.g, if they are synonyms we could replace them by one of them without taking the semantic meaning of the term.

- Document representation: after the above listed "filters", each document is encoded and stored as a standard vector of term weights. 
In the Vector Space Model there are several variations to attribute the weights to the terms. Two of the most common weights are: TF (Term Frequency) and TFIDF (Term Frequency Inverse Term Frequency). We have used TFIDF in our study. [2] provides the following definitions:

$$
T F(\text { term }, \text { document })=\text { the frequencyoftermindocument }
$$

and

$$
I D F=\log \frac{\text { numberofdocumentsincollection }}{\text { numberofdocumentswithterm }}+1 \text {. }
$$

These pre-processing techniques are of utmost importance since they reduce significantly the number attributes that characterise each document attenuating the curse of dimensionality. Stop word removal, stemming and pruning improves classification quality once they remove the meaningless data that leads to a reduction in the number of dimensions in the term space. Document representation concerns the estimation of the importance of a specific term in the document. As the number of features (attributes) are very huge in a collection of documents, the pre-processing techniques help in reducing these huge number of features.

\section{Text Classification}

Text Classification attempts to automatically determine whether a document or part of a document has particular characteristics of interest, usually based on whether the document discusses a given topic or contains a certain type of information [3]. Text Classification involves two main research areas: Information Retrieval and Machine Learning. The first of step of Text Classification is to transform documents into a suitable representation for the Classifier. For this, and before applying the Classifier documents must be pre-processed using Information Retrieval techniques mentioned in the previous section. Some other pre-processing techniques that can be applied to the collection of documents, in order to reduce the huge amount of terms in the collection is called Feature Selection.

A Feature Selection or feature extraction phase is needed to reduce the dimensionality of the document.

There are three Classification techniques: supervised learning, unsupervised learning and semi-supervised learning.

Supervised Learning is based on a training set of examples, where the key idea is to learn from a set of labelled examples (the training set).

In unsupervised learning there is no a priori output. The goal of unsupervised learning is to learn a model that explains well the data. Usually, the result of unsupervised learning is a new explanation or representation of the observation data, which will then lead to improved future decisions.

Semi-supervised learning makes use of both labelled and unlabelled data (typically a small amount of labelled data and a large amount of unlabelled data. Semi-supervised learning [4] [5] is a machine learning paradigm in which the 
model is constructed with a small number of labelled instances and a large number of unlabelled instances. One key idea in semi-supervised learning is to label unlabelled data using certain techniques and thus increase the amount of labelled training data.

\section{Related Work}

The authors in [6] explore the effects of different text representation approaches on the classification performance of MEDLINE documents. In this study the authors use only the title and combine a word representation, the bag-of-words approach with a phrase representation, the bag-of-phrase. Due to previous studies [7] they decided to compare this approach with the bag-of-words representation, bag-of-phrase representation and an hybrid one. They also used the Support Vector Machine Algorithm. The experiments were made with OHSUMED ${ }^{5}$ data set. The authors achieved better performance results with the hybrid approach.

In [8] the authors examined ways to represent text from two aspects related with text representation in a vector space model: (1) what should a term be and (2) how to weight a term. The authors evaluated their approach using a Support Vector Machine algorithm. They found that representing text using a different approaches from the bag-of-words representation does not show performance improvements. They also found that the term weight slightly improved the performance.

In this paper we evaluate the impact of pre-processing techniques ( stop word removal, stemming, WordNet and pruning ) in Classification, that as far as our knowledge have not yet been studied (concerning a MEDLINE data set).

\section{Empirical Study}

Our base of work will be a MEDLINE sample. The focuses of this research is to study the impact of pre-processing techniques in a MEDLINE sample Data Set.

\subsection{Data Set Characterisation}

The data set that is subject of our study, is a MEDLINE sample that was downloaded from the NLM site at (ftp://ftp.nlm.nih.gov/nlmdata/sample/MEDLINE/). This sample has $53.2 \mathrm{MB}$, and contains 30000 citations. The sample is in the XML format. Each citation contains several information namely: the pmid (the PubMed id), the journal title, the PubMed date, the article title, the abstract of the paper if available, the list of authors, the list of keywords and the list of Mesh terms. A MeSH (Medical Subject Headings) is the (U.S.) National Library of

\footnotetext{
${ }^{5}$ which is a database composed of 348,566 MEDLINE documents from 270 journals that are classified under 14,321 mesh categories
} 
Medicine's controlled vocabulary thesaurus. It consists of sets of terms naming descriptors in a hierarchical structure that permits searching at various levels of specificity. A MeSH term is a medical subject heading, or descriptor, as defined in the MeSH thesaurus. We have made a pre-selection of 1098 papers using only the one's containing at least one of the following mesh headings: "Erythrocytes", "Escherichia coli", "Protein Binding" and "Blood Pressure" containing approximately 250 documents per category.

\subsection{Pre-Processing techniques applied}

The MEDLINE sample we used in our study was in the XML format. So the first pre-processing step was to read the XML file and to filter the information we need namely the pmid (PubMed id), journal title, PubMed date, article title, abstract of the paper if available, list of authors, list of keywords and the list of Mesh terms. We have developed our application using the JAVA Programming Language which contains classes to work with XML files and SQL, that are needed for our approach. We have also used a MySQL Database to store all the information useful for further pre-processing and Classification. We have applied the following pre-processing techniques to our original Data Set.

- Tokenization;

- Stop Words removal: we have used a set of 659 stop words;

- We have used WordNet [9] to search for synonyms of terms and replace each term for the respective synonym (we have chosen the smallest one);

- Stemming: we used the Porter's Stemmer Algorithm [10]

- We have implemented the standard term-frequency inverse document frequency (TFIDF) function to assign weights to each term in the document.

Besides this most common pre-processing techniques we have applied some other pre-processing features with the objective of reducing the number of attributes, namely:

- Pruning:

- We have removed bi-grams words that are meaningless (such as "iz", "tk", etc.);

- We have also removed the words that appear less than 10 times in the all collection, because if their frequency is so low probably they are not discriminative of the document content;

- We have also removed the terms that do not appear in the WordNet neither on a medical dictionary of terms (The Hosford Medical Terms Dictionary [11]). The main reason is to eliminate terms that are not valid (such as "tksx") that are meaningless and do not constitute a valid term.

As already mentioned we have used the TFIDF method because in this weighting scheme terms that appear too rarely or too frequently are ranked lower than terms that balance between the two extremes. And also because higher weight terms signify that the term contributes better to classification results.

Figure 1 summarises our approach. 


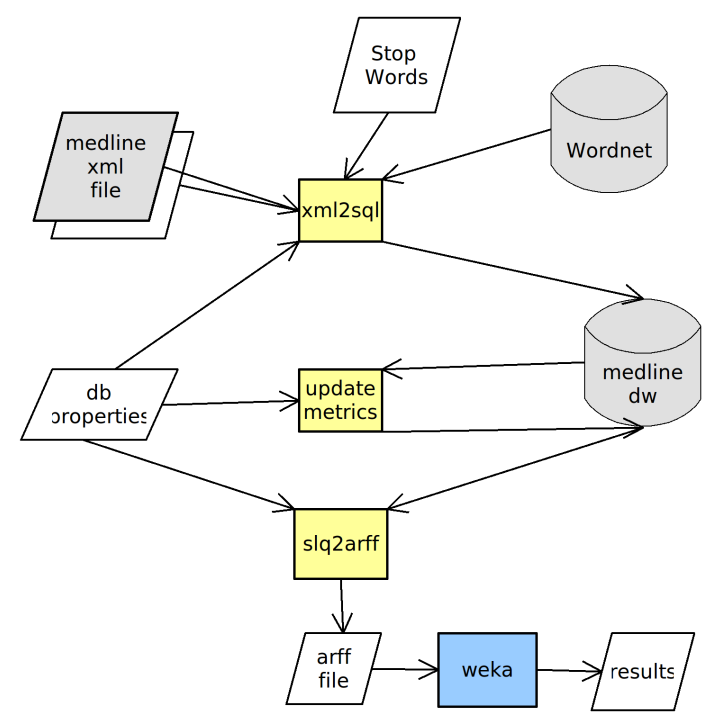

Fig. 1. Summary of steps

\subsection{Data Warehouse Techniques}

Data Warehouse "is a subject-oriented, integrated, non-volatile and time-variant collection of data in support of management's decisions" as defined by [12]. Data Warehouse techniques are used to represent the collection of documents as a set of vectors that can be written as a matrix. A dimensional model comprises facts, dimensions and measures [13]. The fact table represents the measure that is being tracked [14]. In our study was used a Snowflake schema with a central fact table of terms. The ETL process (Extracting, Transforming and Loading and Indexing) was made using a $S Q L$ database. The Extract was made using the $X M L$ source files and populating the $S Q L$ database. The Fact Term table contains measures like the number of occurrence of the term at the title and also at the abstract (representing the $T F=$ Term Frequency). Other measures introduced in the fact table was the $T F * I D F$ (Term Frequency Inverse Document Frequency) to calculate the "Weight" of a term in a document. To calculate these measures, another measure must be used - the Document Frequency - representing the frequency of the term in all the documents collection. This measure can be calculate using the dimension term table. Our model (summarised at Figure 2) includes other dimension tables like a document dimension to represent the citation document; a Time dimension was used to represent the time dimension associated with the citation document; author dimension that have to be represented using a group author due to the $M \longleftrightarrow M$ relation between the term fact table and the authors dimension. Using this techniques, the calculation process that is represented by the "update metrics" in Figure 1 perform the final calculations and was made using only $S Q L$ instructions. With this repre- 
sentation, the creation of the data set is simplified, since it can be done using $S Q L$ instructions to join the records, according to our needs to produce the output data set. Also, the pruning can be made with $S Q L$ instructions that limit the number of terms that should be used. The last step is represented by the "sql2arff" process in Figure 1 and was applied the following pruning technique through our Data Warehouse:

$-<10 *$ To remove words that appear less than 10 times in the document collection.

$-<100 *$ To remove words that appear less than 100 times in the document collection.

All the $S Q L$ instruction used by our Java programs are on the db.properties file in Figure 1. If we decide to change the Mesh, the pruning to apply or any other characteristics we simple need to change this file.

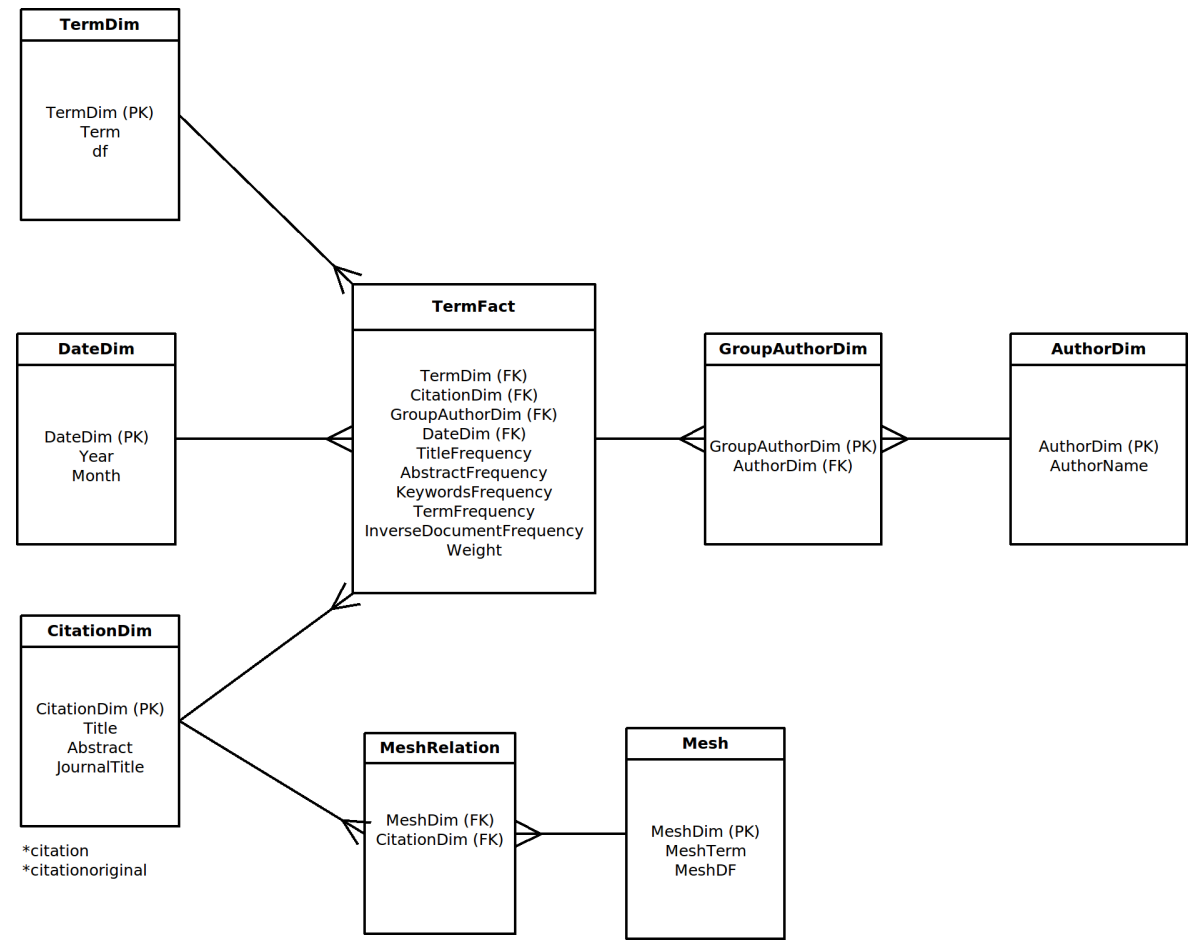

Fig. 2. Snowflake Schema

With this technique was possible to reduce the number of attributes from more than 1300 to less then 90 . This can have a significantly impact in processing the MEDLINE database. 


\section{$6 \quad$ Experimental Results}

Table 1 lists the algorithms available in WEKA and used in our experiments. We have generated several data sets combining the different pre-processing techniques (pruning, stemming and WordNet). The quality of the classifiers were assessed by their Accuracy.

Table 1. Machine Learning algorithms used in the study. RF stands for Random Forest.

\begin{tabular}{|ll|}
\hline Algorithm & \multicolumn{1}{|c|}{ Type } \\
\hline SMO & Support Vector Machine \\
RF & Ensemble algorithm \\
IBk & K-nearest neighbours \\
BayesNet & Bayes Network \\
j48 & Decision tree \\
dtnb & Decision table/naive bayes hybrid \\
\hline
\end{tabular}

The results obtained are in Table 2. In the Pruning column we present the thresholds for term occurrence (terms that appear less than 10 and less than 100 times (df) in the document collection and that were removed).

Table 2. Accuracy Classification Results. The majority class is $28 \%$.

\begin{tabular}{|l|c|c|r|r|r|r|r|r|r|}
\hline Pruning & Stemming & WordNet & Attributes & \multicolumn{5}{|c|}{ Acc } \\
& & & & SMO & RF & IBk & BayesNet & j48 & dtnb \\
\hline$<10 *$ & no & no & 1304 & 76.95 & 84.09 & 46.66 & 86.85 & 81.33 & 76.28 \\
\hline$<100 *$ & no & no & 62 & 76.62 & 81.70 & 63.02 & 81.60 & 80.74 & 77.01 \\
\hline$<10 *$ & yes & no & 1103 & 80.57 & 84.85 & 49.71 & 86.85 & 84.57 & 80.57 \\
\hline$<100 *$ & yes & no & 90 & 77.31 & 85.51 & 61.10 & 85.03 & 84.17 & 79.88 \\
\hline$<10 *$ & no & yes & 916 & 76.38 & 84.95 & 48.19 & 87.14 & 83.90 & 79.42 \\
\hline$<100 *$ & no & yes & 85 & 80.24 & 85.20 & 61.45 & 85.97 & 83.77 & 79.77 \\
\hline$<10 *$ & yes & yes & 904 & 78.00 & 85.14 & 51.42 & 87.42 & 83.71 & 80.66 \\
\hline$<100 *$ & yes & yes & 91 & 77.71 & 85.80 & 57.33 & 86.38 & 82.66 & 80.47 \\
\hline
\end{tabular}

The first experiences shows that the application of pruning, stemming and WordNet reduces significantly the number of attributes without affecting the accuracy of results. We get the best results using the Random Forest $(85.80 \%)$ using a pruning of $d f=100$, using the stemming and the WordNet. The accuracy result are even best with only 91 terms than using 904 terms. The Bayes Network algorithm with a pruning of $d f=10$, with Stemming and WordNet show the best result, but if we look at the result of using pruning with $d f=100$ the accuracy drop from $87.42 \%$ to $86.38 \%$. 
The time taken to build the models are presented on in Table 3.

Table 3. Time to construct the models in seconds

\begin{tabular}{|l|c|c|r|r|r|r|r|r|r|}
\hline Pruning & Stemming & WordNet & Attributes & \multicolumn{6}{|c|}{ Time } \\
& & & SMO & RF & IBk & BayesNet & j48 & dtnb \\
\hline$<10 *$ & no & no & 1304 & 1.32 & 101.98 & 0.01 & 2.5 & 8.16 & 34.05 \\
\hline$<100 *$ & no & no & 62 & 0.8 & 2.39 & 0.01 & 0.38 & 0.56 & 3.19 \\
\hline$<10 *$ & yes & no & 1103 & 1.27 & 69.69 & 0.01 & 2.03 & 10.55 & 54.64 \\
\hline$<100 *$ & yes & no & 90 & 0.75 & 2.39 & 0.02 & 0.43 & 1.17 & 5.96 \\
\hline$<10 *$ & no & yes & 916 & 1.45 & 61.23 & 0.01 & 1.74 & 7.81 & 58.78 \\
\hline$<100 *$ & no & yes & 85 & 1.25 & 3.14 & 0.01 & 0.8 & 1.4 & 3.68 \\
\hline$<10 *$ & yes & yes & 904 & 1.77 & 59.97 & 0.01 & 1.6 & 8.16 & 34.05 \\
\hline$<100 *$ & yes & yes & 91 & 1.3 & 3.96 & 0.04 & 0.74 & 1.24 & 5.56 \\
\hline
\end{tabular}

The time taken to build the models were obtained on a intel Core 2 Duo Processor @ 2.40Ghz with 4096 GB. We can see that pruning has a positive impact in the time taken to build the models specially on algorithms that take longer to execute.

\section{Conclusions and Future Work}

This paper focuses on the study of the impact of pre-processing techniques in classifying MEDLINE documents. We have presented results of our empirical study of the impact of the pre-processing techniques in text classification. The amount of information available on the MEDLINE database can (and probably will) be an issue. Through the use of Information Retrieval and Data Warehouse techniques applied it was possible to reduce significantly the time needed for the pre-processing without affecting the accuracy. Although we are using a MEDLINE sample we have a data set with 30000 MEDLINE citations and we selected only 1098 citations with abstract representing the 4 classes using only the one's containing at least one of the following mesh headings: "Erythrocytes", "Escherichia coli", "Protein Binding" and "Blood Pressure". In this sample we started with more than 1300 attributes that can be represented using a fact table and some dimensions tables in our Snowflake schema data warehouse. The pruning pre-processing was made using only $S Q L$ instructions on the Data Warehouse. Unlike [6] we also have used the abstract from the papers. Like [8] we have used a bag-of-words representation and we implemented the standard term-frequency inverse document frequency (TFIDF) function to assign weights to each term. We have generated several data sets combining the different pre-processing techniques. We have made a comparison table of the accuracy obtained using different pre-processing techniques and different classification algorithms. We have also presented the computation time required for 
the execution of the algorithms. The best accuracy result achieved $(87.42 \%)$ is thus promising.

As a future work and to achieve a better classification we may: 1) incorporate more information 2) optimise the MeSH terms selection for each document 3) test with other MEDLINE sources, like the 2010 MEDLINE version and 4) using the full MEDLINE database.

\section{References}

1. Wheeler, D.L., Barrett, T., Benson, D.A., Bryant, S.H., Canese, K., Chetvernin, V., Church, D.M., Dicuccio, M., Edgar, R., Federhen, S., Geer, L.Y., Helmberg, W., Kapustin, Y., Kenton, D.L., Khovayko, O., Lipman, D.J., Madden, T.L., Maglott, D.R., Ostell, J., Pruitt, K.D., Schuler, G.D., Schriml, L.M., Sequeira, E., Sherry, S.T., Sirotkin, K., Souvorov, A., Starchenko, G., Suzek, T.O., Tatusov, R., Tatusova, T.A., Wagner, L., Yaschenko, E.: Database resources of the national center for biotechnology information. Nucleic Acids Res 34 (2006)

2. Zhou, W., Smalheiser, N.R., Yu, C.: A tutorial on information retrieval: basic terms and concepts. Journal of Biomedical Discovery and Collaboration 1 (2006)

3. Cohen, A.M., Hersh, W.R.: A survey of current work in biomedical text mining. Briefings in Bioinformatics 6 (2005) pp. 57-71

4. Chapelle, O., et al.: Semi-supervised learning. Cambridge MIT Press (2006)

5. Zhu, X.: Semi-supervised learning literature survey (2006)

6. Meliha Yetisgen-Yildiz, W.P.: The effect of feature representation on medline document classification. AMIA Annu Symp Proc. (2005) 849-853

7. Sebastiani, F.: Machine learning in automated text categorization. Computing Surveys. 31(1) (2002) 1-47

8. LAN, M., TAN, C.L., SU, J., LOW, H.B.: Text representations for text categorization : a case study in biomedical domain. In: IJCNNâ07 : International Joint Conference on Neural Networks. (2007)

9. Miller, G.A.: Wordnet: A lexical database for english. Communications of the ACM 38 (1995) pp. 39-41

10. Porter, M.F.: An algorithm for suffix stripping (1997)

11. : Hosford medical terms dictionary v3.0 (2010)

12. Inmon, W.: Building the Data Warehouse. . (2002)

13. Schonbach, C., Kowalski-Saunders, P., Brusic, V.: Data warehousing in molecular biology. Briefings in Bioinformatics 1 (2000) 190-198

14. Chituc, C.M.: Data warehousing - lecture notes (2009) 